\title{
Remark to Response of A. V. Lichtenstein
}

\author{
V. N. Manskikh \\ Lomonosov Moscow State University, Faculty of Bioengineering and Bioinformatics, \\ 119991 Moscow, Russia; E-mail: manskikh@mail.ru
}

Received October 8, 2016

\begin{abstract}
The arguments against the "bad luck" hypothesis suggested by C. Tomasetti and B. Vogelstein ((2015) Science, $\mathbf{3 4 7}, \mathbf{7 8 - 8 1 )}$ are significant in any case, and new experimental studies are necessary.
\end{abstract}

DOI: $10.1134 / \mathrm{S} 0006297917010114$

Keywords: cancer, "bad luck" hypothesis, carcinogenesis, mutagenesis, extrinsic and intrinsic factors, cancer prevention

First, I would like to note that my comment [1], as it follows from its title, suggests or at least admits a somewhat wider discussion of the problem than only consideration of statements in the work by A. V. Lichtenstein [2]. In this connection, it is hardly reasonable the reproach that the comment concerns weak points of the work by $\mathrm{C}$. Tomasetti and B. Vogelstein [3] (initiating the polemics about extrinsic and intrinsic factors) which were not given sufficient attention by Lichtenstein. If some of these notes were not spoken out earlier (however, some of them have been spoken out), it is not evidence of their insignificance.

I would not like to enter the polemics about "methodological bases" of modern biology, but it is obvious that the creation of a "theoretical hybrid" from data on rodents' stem cells and frequencies of human tumors - is very different from using appropriate model organisms for investigating general biological laws. At least, because of the pronounced difference in spectra of human and rodent tumor frequencies.

It seems strange that the opponent thinks foggy or unrelated to the problem under discussion some aspects concerned (sometimes not in detail) in his own paper (epidemiological data indicating the absence of a decrease in cancer frequency and presented in my comment with the simplest mathematical symbols; the endogenous origin of spontaneous tumors in animals; cancer-restraining mechanisms in situ, etc.). The same may be said about external uncontrolled factors which, despite the statement of Lichtenstein [2], are known (therefore, they are not liable to removing with "Occam's razor"), except the impossibility (for the time being!) of accurate measurement of their contribution to carcinogenesis. By the way, these factors are mentioned in the opponent's work, but he does not take this impossibility into account in his further argumentation.
Really, molecular signatures would be the best tool for verification of uncontrolled factors of carcinogenesis when epidemiology is impotent. However, by now, molecular signatures are not studied sufficiently; moreover, the literature presents an interpretation of these data that is opposite to that proposed by the opponent; some signatures are consequences of defects in DNA repair, and such signatures can be called "endogenous mutations" only with reservations. Therefore, the data presented on signatures cannot be a decisive argument at this time. All these points are obvious from the signature problem description in Lichtenstein's work, and I thought and think now that it is not necessary to comment on them additionally.

However, it is quite reasonable to agree with the opponent that just the readers will judge about the persuasiveness of the arguments presented and that the fairness of one of the alternative concepts (or of their synthetic variant proposed by Lichtenstein) will be established by further studies. The appearance of the first prominent experimental work on the problem discussed can be only awaited.

\section{REFERENCES}

1. Manskikh, V. N. (2017) Do external or internal factors lead to tumor development? It is still unknown, Biochemistry (Moscow), 82, 81-85.

2. Lichtenstein, A. V. (2017) Response to Comments by V. N. Manskikh: "Do external or internal factors lead to tumor development? It is still unknown", Biochemistry (Moscow), 82, 86-87.

3. Tomasetti, C., and Vogelstein, B. (2015) Cancer etiology. Variation in cancer risk among tissues can be explained by the number of stem cell divisions, Science, 347, 78-81. 